\title{
Job Crafting: Conceituação e Qualidade Científica das Medidas
}

\author{
Rita Pimenta de Devotto ${ }^{1}$, Solange Muglia Wechsler \\ Pontifícia Universidade Católica de Campinas, Campinas-SP, Brasil
}

\section{RESUMO}

O job crafting é um processo de ações proativas individuais para redesenhar o trabalho, visando adequá-lo aos valores, competências e motivações individuais, ou para otimizar recursos e demandas com necessidades e recursos pessoais. Verificou-se as qualidades científicas das medidas do construto em quatro bases de dados no período entre 2001 a agosto de 2017. Identificou-se duas formas diferentes de conceitualizar o construto, quatro instrumentos diferentes para sua avaliação e sete trabalhos de adaptação e validação para outros contextos. Analisou-se os 11 instrumentos selecionados em termos de fontes de evidência de validade e precisão. As formas mais utilizadas foram as evidências de validade baseadas na estrutura interna e na relação com variáveis externas e a precisão por meio do alfa de Cronbach. Duas medidas foram validadas para o Brasil, possibilitando a pesquisa sobre as ações de redesenho do trabalho. Conclui-se que o job crafting é mensurável de forma válida e precisa.

Palavras-chave: redesenho do trabalho; avaliação psicológica; validade; precisão; comportamento organizacional.

\section{ABSTRACT - Job Crafting: Conceptualization and Scientific Quality of the Measures}

Job crafting is a process of proactive individual actions to redesign work to match individual values, competencies and motivations, or to optimize resources and demands with personal needs and resources. We verified the scientific qualities of the construct measures in four databases between 2001 and August 2017. We identified two different ways of conceptualizing the construct, four different instruments for its evaluation, and seven works to adapt and validate for other contexts. We analyzed 11 selected instruments in terms of validity evidence and accuracy. The most used forms were those with validity evidence based on the internal structure, and those that related external variables and accuracy through Cronbach's alpha. Two measures were validated for Brazil, enabling research on work redesign actions, and we concluded that job crafting is measurable in validity and accuracy.

Keywords: work redesign; psychological evaluation; validity; accuracy; organizational behavior.

\section{RESUMEN - Job Crafting: Concepción y Calidad Científica de las Medidas}

El Job crafting es un proceso de acciones proactivas individuales para rediseñar el trabajo con el fin de adecuarlo a los valores, competencias y motivaciones individuales, o para optimizar recursos y demandas con necesidades y recursos personales. Verificamos las cualidades científicas de las medidas del constructo en cuatro bases de datos en el período de 2001 a agosto de 2017. Identificamos dos formas diferentes de conceptualizar el constructo, cuatro instrumentos diferentes para su evaluación y siete trabajos de adaptación y validación para otros contextos. Analizamos los 11 instrumentos seleccionados en términos de fuentes de evidencia de validez y precisión. Las formas más utilizadas fueron las evidencias de validez basadas en la estructura interna y en la relación con variables externas y la precisión por medio del alpha de Cronbach. Dos medidas fueron validadas para Brasil posibilitando la investigación sobre las acciones de rediseño del trabajo. Se concluye que el job crafting es mensurable de forma válida y precisa.

Palabras clave: rediseño del trabajo; evaluación psicológica; validez; precisión; comportamiento organizacional.

O job crafting é um construto que se refere às mudanças físicas e cognitivas realizadas pelo indivíduo para modificar as tarefas e as relações no trabalho (Wrzesniewski \& Dutton, 2001). O termo foi cunhado para capturar as ações proativas ascendentes que modificam o delineamento do trabalho. Segundo esse conceito, os indivíduos podem moldar o próprio trabalho alinhando-o aos seus valores, paixões e competências individuais para tornar seu trabalho mais envolvente e gratificante (Berg, Dutton, \& Wrzesniewski, 2008).

A terminologia "redesenho do trabalho" (Chinelato, Ferreira, \& Valentini, 2015) tem sido utilizada em português para fazer referência ao job crafting. $\mathrm{O}$ redesenho do trabalho se alinha teoricamente ao campo de estudo do job design, que investiga como "trabalhos, tarefas e papéis são estruturados, postos em prática e modificados e qual o impacto destas estruturas, 
realizações e modificações nos resultados individuais, grupais e organizacionais" (Grant \& Parker, 2009, p. 5). Nessa perspectiva, o redesenho do trabalho compreende as ações individuais ascendentes que modificam as características e o ambiente social do trabalho. Tais ações têm o potencial de complementar os delineamentos tradicionais descendentes e podem resolver inadequações entre indivíduo - posto de trabalho (Demerouti, 2014).

De acordo com Wrzesniewski, LoBuglio, Dutton e Berg (2013), o redesenho do trabalho pelo indivíduo engloba três tipos de estratégias: ações de redesenho da tarefa (task crafting), as reformulações cognitivas (cognitive crafting) e as ações de redesenho das relações (relational crafting). A primeira estratégia inclui mudanças tangíveis no conjunto de tarefas que o indivíduo considera ser seu trabalho (e.g., número, escopo e tipo de tarefa). As reformulações cognitivas envolvem mudanças no significado e propósito atribuídos pelo indivíduo ao seu trabalho (e.g., percepção do trabalho não apenas como um conjunto de tarefas concatenadas, mas como parte significativa de um todo). A terceira estratégia engloba mudanças na quantidade e na qualidade das interações no trabalho (com colegas, superiores, clientes, fornecedores). O redesenho proativo do trabalho acontece em todos os tipos de trabalho, independentemente do grau de autonomia, complexidade e autoridade envolvidos (Wrzesniewski et al., 2013).

Posteriormente, Tims e Bakker (2010), interessados em capturar os comportamentos efetivos e as mudanças tangíveis que os indivíduos utilizam para redesenhar seu trabalho, propuseram o enquadramento do job crafting ao modelo de Recursos e Demandas no TrabalhoRDT (Bakker \& Demerouti, 2007). O RDT específica como o bem-estar e o desempenho no trabalho podem ser produzidos pelo equilíbrio entre os recursos do trabalho (fatores motivacionais) e as demandas de trabalho (fatores de desgaste). De acordo com os autores, o job crafting seria uma forma específica de comportamento proativo na qual o indivíduo inicia mudanças no nível das demandas e dos recursos de trabalho para lograr um equilíbrio entre ambos e suas necessidades e recursos pessoais (Tims \& Bakker, 2010). As ações de redesenho do trabalho foram propostas em três dimensões: aumentar os recursos do trabalho, aumentar as demandas de trabalho desafiadoras e diminuir as demandas de trabalho que impedem o alcance dos resultados individuais (Tims \& Bakker, 2010). Nessa proposição teórica, avalia-se as mudanças tangíveis na tarefa e nas relações de trabalho e desconsidera-se a dimensão da reformulação cognitiva (cognitive crafting), dado que esta implica em mudanças intangíveis na percepção do indivíduo sobre seu trabalho (Lichtenthaler \& Fischbach, 2016; Tims \& Bakker, 2010).

Desde o surgimento do construto em 2001 até o ano de 2009, as pesquisas sobre job crafting foram essencialmente qualitativas ou de natureza teórica (Berg, Grant, \& Johnson, 2010; Berg, Wrzesniewski \& Dutton, 2010). A partir de 2012, constatou-se um crescimento constante de publicações científicas sobre o construto, especificamente de estudos empíricos quantitativos, motivados pelo desenvolvimento de medidas. Evidenciou-se uma difusão das publicações em diferentes periódicos (e.g., áreas de psicologia organizacional, comportamento organizacional, negócios e recursos humanos), liderada pela Holanda (Petrou, Demerouti, Peeters, Schaufeli, \& Hetland, 2012; Tims \& Bakker, 2012; Tims, Bakker, \& Derks, 2014). Evidenciou-se a inserção da Ásia (China, Taiwan e Japão) no cenário de produção científica internacional sobre job crafting, e ausência de autores com filiação em países latino-americanos, inclusive o Brasil (Pimenta de Devotto, \& Machado, 2017). Entre 2012 e maio de 2015, houve uma predominância de estudos empíricos (73,5\%), em relação aos estudos teóricos (26,5\%), sendo que $80,0 \%$ dos estudos empíricos foram quantitativos e valeram-se sobretudo de duas escalas para aferir o construto em populações ocupacionais gerais (Pimenta de Devotto \& Machado, 2017): a Job Crafting Scale - JCS (Tims, Bakker, \& Derks, 2012) e o Job Crafting Questionnaire JCQ (Slemp \& Vella-Brodrick, 2013).

Portanto, a partir do trabalho seminal de Wrzesniewski \& Dutton (2001), a pesquisa empírica sobre os antecedentes, desfechos e correlatos do redesenho do trabalho cresceu rapidamente na última década. Esse aumento foi devido, em grande parte, à aceitação do modelo teórico de Tims e Bakker (2010) e à operacionalização do construto em termos do modelo RDT (Tims et al., 2012), sendo essa perspectiva do job crafting a mais comumente utilizada nas pesquisas internacionais (Rudolph, Katz, Lavigne, \& Zacher, 2017). Após o estágio inicial de desenvolvimento de medidas a partir dos dois modelos teóricos (Tims \& Bakker, 2010; Wrzesniewski \& Dutton, 2001), a pesquisa na área tem produzido estudos de adaptação transcultural das medidas originais para outros idiomas e contextos de forma a poder avançar o conhecimento sobre o redesenho do trabalho em diferentes realidades. Recentemente, estudos sobre intervenções que estimulam os comportamentos de redesenho do trabalho (job crafting interventions) também ganharam terreno e impulso na pesquisa (Bakker, 2015). Logo, esse cenário de expansão da pesquisa sobre o redesenho do trabalho, de refinamento e adaptação transcultural dos instrumentos utilizados para avaliar o construto, justifica uma revisão sobre a qualidade científica das medidas produzidas até o momento.

O critério contemporâneo de qualidade científica dos instrumentos abrange a busca por diversas fontes de evidências de validade e precisão. O termo "validade" se refere à adequação e à utilidade de um instrumento para um propósito específico (Geisinger, 2013). As diretrizes 
da literatura (American Educational Research Association [AERA], American Psychological Association [APA] \& National Council on Measurement in Education [NCME], 2014), especificam cinco tipos de fontes de evidências de validade: baseadas no conteúdo, no processo de resposta, na estrutura interna, nas relações com variáveis externas e nas consequências da testagem. A precisão é considerada uma precondição para a validade, indica a consistência da medida e revela a confiabilidade nos resultados obtidos. A precisão pode ser estimada por meio de quatro tipos de métodos: por estabilidade, por equivalência, pela consistência interna e pela generalização (Geisinger, 2013). Até a presente data, não foram localizados estudos de revisão que analisaram a existência de tais qualidades científicas nos instrumentos que avaliam o job crafting.

Portanto, o objetivo deste artigo é investigar as qualidades psicométricas das escalas destinadas a medir o construto do job crafting (redesenho do trabalho) na literatura internacional e no Brasil. Com base nas diretrizes da literatura (AERA, APA, \& NCME, 2014) serão descritas quais as fontes de evidências de validade e precisão foram investigadas nos estudos sobre medidas de job crafting.

\section{Método}

\section{Amostra}

Utilizou-se o portal de Periódicos da Coordenação de Aperfeiçoamento de Pessoal de Nível Superior CAPES para efetuar uma busca em três bases de dados internacionais: PsycINFO (American Psychological Association), Academic Search Premier - ASP (EBSCOhost) e Directory of Open Access Journals - DOAJ, no período compreendido entre 2001 até agosto de 2017. Esta revisão utilizou como descritores: "job crafting" e sua aparição no campo "abstract", o operador booleano "AND", e o descritor "validity" e sua aparição nos campos "any field" (PsycINFO) e "texto completo" (ASP). Na base de dados DOAJ foram utilizados os mesmos descritores "job crafting AND validity" em seu campo único de busca. Conjuntamente a essa busca em bases internacionais, o Banco de Teses da CAPES foi acessado, utilizando os descritores "job crafting AND validity", com o objetivo de recuperar dissertações e teses produzidas no Brasil sobre medidas de job crafting.

\section{Procedimento}

Retornaram 29 artigos revisados por pares e uma dissertação (30 trabalhos) das bases de dados consultadas. Foram excluídos desta revisão os artigos revisados por pares sem texto completo disponível em língua inglesa $(n=1)$ e eliminamos as duplicadas $(n=5)$, resultando um total de 24 trabalhos. Posteriormente, uma triagem foi realizada a partir da leitura dos resumos desses 24 trabalhos. Buscou-se identificar aquelas publicações que efetivamente relacionavam-se à construção de medidas de job crafting e à adaptação e validação de medidas existentes para outros contextos. Dos 24 trabalhos, 14 foram excluídos por não atenderem a esses critérios seleção. Dessa forma, 10 artigos revisados por pares e uma dissertação foram selecionados. Por fim, os 11 trabalhos selecionados foram analisados segundo as diretrizes da literatura, sendo as propriedades psicométricas dos instrumentos identificados avaliadas de acordo com as fontes de evidências de validade e precisão (AERA, APA, \& NCME, 2014).

\section{Resultados e Discussão}

A Tabela 1 apresenta as quatro medidas de job crafting identificadas. Três medidas avaliam o construto em populações ocupacionais gerais (Niessen, Weseler, \& Kostova, 2016; Slemp \& Vella-Brodrick, 2013; Tims, Bakker, \& Derks, 2012) e uma medida em trabalhadores operacionais (Nielsen \& Abildgaard, 2012). Observa-se também a existência de duas conceitualizações sobre o job crafting: (a) a proposta por Wrzesniewski e Dutton (2001) e (b) a proposta por Tims e Bakker (2010). Ambas formas de conceituar o construto produziram diferentes formas de avaliar as suas dimensões.

A Tabela 2 apresenta os estudos de adaptação das quatro medidas originais para outros contextos. Destas quatro medidas originais de job crafting, três medidas (Nielsen \& Abildgaard, 2012; Slemp \& Vella-Brodrick, 2013; Tims et al., 2012) foram adaptadas para outros idiomas e contextos, em alguns casos replicando a estrutura fatorial original das escalas adaptadas. Observase que a Job Crafting Scale (Tims et al., 2012) foi a escala que mais produziu estudos $(n=5)$ de adaptação e validação para outros contextos (África do Sul, Alemanha, Brasil, Itália e Japão).

A Tabela 3 sumariza os resultados da análise sobre as fontes de evidências de validade e precisão de cada uma das 11 medidas identificadas. Observa-se que todos os estudos de adaptação e validação buscaram fontes de evidências de validade baseados na estrutura interna. Com relação as variáveis externas, o job crafting foi avaliado em relação a vários antecedentes individuais (e.g., personalidade proativa, inciativa pessoal, necessidades psicológicas básicas, autoeficácia, capital psicológico positivo) e aos seus antecedentes contextuais (e.g., autonomia, demandas e recursos do trabalho). Avaliou-se também o job crafting em relação aos seus desfechos positivos de bem-estar ocupacional (e.g., engajamento no trabalho, satisfação no trabalho, afetos positivos) e de desempenho; e em relação aos desfechos negativos (e.g., burnout, afeto negativo). As fontes de precisão foram investigadas principalmente pelo alfa de Cronbach. 
Tabela 1

Job Crafting: Formulação Teórica e Dimensões do Conceito

\begin{tabular}{|c|c|c|c|c|}
\hline Autor/Ano/Medida & $\begin{array}{l}\text { Formulação } \\
\text { teórica }\end{array}$ & $\begin{array}{l}\text { Dimensões do } \\
\text { Job crafting }\end{array}$ & $\begin{array}{c}\text { país/ } \\
\text { amostra }\end{array}$ & $\begin{array}{l}\text { Adaptação } \\
\text { e Validação } \\
\text { para outros } \\
\text { países }\end{array}$ \\
\hline $\begin{array}{l}\text { Tims, Bakker, } \\
\text { \& Derks (2012) } \\
\text { Job Crafting Scale (JCS) }\end{array}$ & $\begin{array}{c}\text { Tims \& } \\
\text { Bakker (2010) }\end{array}$ & $\begin{array}{l}\text { Aumentando os recursos estruturais do trabalho }{ }^{1} \\
\text { Diminuindo as demandas de obstáculo } \\
\text { Aumentando os recursos sociais do trabalho } \\
\text { Aumentando as demandas de trabalho desafiadoras }\end{array}$ & $\begin{array}{l}\text { Holanda, } \\
\mathrm{N}=1.181\end{array}$ & $\begin{array}{l}\text { África do Sul } \\
\text { Alemanha } \\
\text { Brasil } \\
\text { Itália } \\
\text { Japão }\end{array}$ \\
\hline $\begin{array}{l}\text { Nielsen \& } \\
\text { Abildgaard (2012) } \\
\text { Job Crafting } \\
\text { Questionnaire - Blue- } \\
\text { collar workers (JCRQ) }\end{array}$ & $\begin{array}{c}\text { Tims \& } \\
\text { Bakker (2010) }\end{array}$ & $\begin{array}{l}\text { Aumentando as demandas de trabalho desafiadoras } \\
\text { Diminuindo as demandas sociais de trabalho } \\
\text { Aumentando os recursos estruturais do trabalho } \\
\text { Aumentando as demandas quantitativas } \\
\text { Diminuindo as demandas de obstáculo }\end{array}$ & $\begin{array}{l}\text { Dinamarca, } \\
\mathrm{N}=770\end{array}$ & $\begin{array}{l}\text { Espanha } \\
\text { Reino Unido } \\
\text { China } \\
\text { Taiwan }\end{array}$ \\
\hline $\begin{array}{l}\text { Slemp \& } \\
\text { Vella-Brodrick (2013) } \\
\text { Job Crafting } \\
\text { Questionnaire (JCQ) }\end{array}$ & $\begin{array}{l}\text { Wrzesniewski } \\
\text { \& Dutton } \\
\text { (2001) }\end{array}$ & $\begin{array}{l}\text { Redesenho da tarefa }{ }^{7} \\
\text { Reformulação cognitiva } \\
\text { Redesenho das relações }\end{array}$ & $\begin{array}{l}\text { Austrália, } \\
\text { N=470 }\end{array}$ & Brasil \\
\hline $\begin{array}{l}\text { Niessen, Weseler, } \\
\text { \& Kostova (2016) } \\
\text { Job Crafting Scale (JCS) }\end{array}$ & $\begin{array}{l}\text { Wrzesniewski } \\
\text { \& Dutton } \\
\text { (2001) }\end{array}$ & $\begin{array}{l}\text { Redesenho da tarefa } \\
\text { Reformulação cognitiva }^{8} \\
\text { Redesenho das relações }^{9}\end{array}$ & $\begin{array}{l}\text { Alemanha, } \\
\mathrm{N}=584\end{array}$ & \\
\hline
\end{tabular}

Tabela 2

Adaptação de Medidas de Job Crafting para outros Contextos

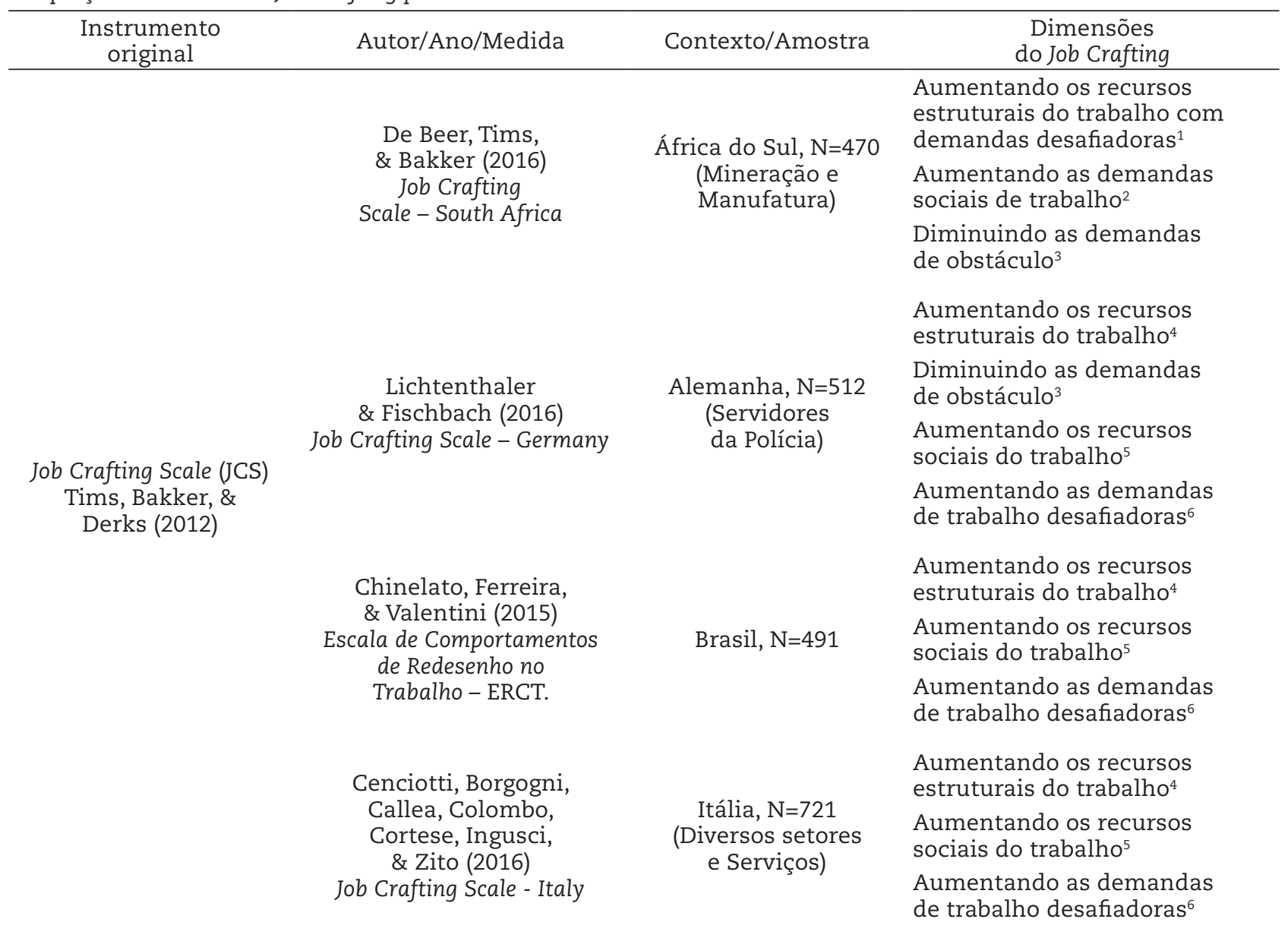


Tabela 2 (continuação)

Adaptação de Medidas de Job Crafting para outros Contextos

\begin{tabular}{|c|c|c|c|}
\hline $\begin{array}{c}\text { Instrumento } \\
\text { original }\end{array}$ & Autor/Ano/Medida & Contexto/Amostra & $\begin{array}{c}\text { Dimensões } \\
\text { do Job Crafting }\end{array}$ \\
\hline \multirow{4}{*}{$\begin{array}{c}\text { Job Crafting Scale (JCS) } \\
\text { Tims, Bakker, \& } \\
\text { Derks (2012) }\end{array}$} & \multirow{4}{*}{$\begin{array}{c}\text { Eguchi, Shimazu, } \\
\text { Bakker, Tims, Kamiyama, } \\
\text { Hara, \& Kawakami (2016) } \\
\text { Job Crafting Scale - JCS-J }\end{array}$} & \multirow{4}{*}{$\begin{array}{l}\text { Japão, N=972 } \\
\text { (Manufatura) }\end{array}$} & $\begin{array}{l}\text { Aumentando os recursos } \\
\text { estruturais do trabalho }\end{array}$ \\
\hline & & & $\begin{array}{l}\text { Diminuindo as demandas } \\
\text { de obstáculo } 3\end{array}$ \\
\hline & & & $\begin{array}{l}\text { Aumentando os recursos } \\
\text { sociais do trabalho }\end{array}$ \\
\hline & & & Aumentando as demandas \\
\hline
\end{tabular}

de trabalho desafiadoras ${ }^{6}$

\author{
Job Crafting \\ Questionnaire for \\ Blue Collars (JCRQ) \\ Nielsen \& Abildgaard \\ (2012) \\ Nielsen, Antino, \\ Sanz-Vergel, \& \\ Rodríguez-Muñoz (2017) \\ Job Crafting \\ Questionnaire - JCRQ
}

Aumentando as demandas de trabalho desafiadoras ${ }^{5}$

Diminuindo as demandas sociais de trabalho ${ }^{7}$

\section{Aumentando os recursos sociais do trabalho ${ }^{5}$ \\ Aumentando as demandas quantitativas $^{8}$ \\ Diminuindo as demandas de obstáculo ${ }^{3}$ \\ Espanha, $\mathrm{N}=164$ \\ China, $\mathrm{N}=170$ \\ Taiwan, $\mathrm{N}=165$}
Pimenta de Devotto
\& Machado (2017)
Escala de Ações
de Redesenho no

\author{
Brasil, $\mathrm{N}=473$ \\ (Diversos \\ setores)
}

\section{Redesenho da tarefa ${ }^{9}$ Reformulações cognitivas ${ }^{10}$ Redesenho das relações ${ }^{11}$}

Trabalho - EART

Nota. Dimensões do Job crafting no original em língua inglesa: ${ }^{1}$ Increasing structural job resources with challenging demands; ${ }^{2}$ Increasing social job demands; ${ }^{3}$ Decreasing hindering job demands; ${ }^{4}$ Increasing structural job resources; ${ }^{5}$ Increasing social job resources; ${ }^{6}$ Increasing challenging job demands; ${ }^{7}$ Decreasing social job demands; ${ }^{8}$ Increasing quantitative demands; ${ }^{9}$ Task crafting; ${ }^{10}$ Cognitive crafting;

${ }^{11}$ Relational crafting

Tabela 3

Qualidades Psicométricas das Medidas de Job Crafting nos Estudos Encontrados

\begin{tabular}{|c|c|c|c|}
\hline Escala - Autoria - País & $\begin{array}{c}\text { Estrutura } \\
\text { interna }\end{array}$ & $\begin{array}{c}\text { Fontes de evidências de validade } \\
\text { Relação com variáveis externas }\end{array}$ & $\begin{array}{l}\text { Fontes de } \\
\text { precisão }\end{array}$ \\
\hline $\begin{array}{l}\text { 1.0. Job Crafting Scale - JCS (Holanda) } \\
\text { Tims, Bakker, \& Derks (2012) }\end{array}$ & $\mathrm{x}$ & $\begin{array}{l}\text { Personalidade proativa, iniciativa pessoal, } \\
\text { avaliação de colegas sobre o engajamento } \\
\text { e desempenho no trabalho. }\end{array}$ & $\begin{array}{c}\text { Alfa } \\
\text { Cronbach }\end{array}$ \\
\hline $\begin{array}{l}\text { 1.1. Job Crafting Scale - JCS (África do Sul) } \\
\text { de Beer, Tims, \& Bakker (2016) }\end{array}$ & $\mathrm{x}$ & $\begin{array}{l}\text { Engajamento no trabalho e } \\
\text { satisfação no trabalho. }\end{array}$ & $\begin{array}{l}\text { Coeficiente } \\
\text { ômega }\end{array}$ \\
\hline $\begin{array}{l}\text { 1.2. Job Crafting Scale - JCS (Alemanha) } \\
\text { Lichtenthaler \& Fischbach (2016) }\end{array}$ & $\mathrm{x}$ & $\begin{array}{l}\text { Iniciativa pessoal, controle do trabalho, } \\
\text { engajamento no trabalho e exaustão emocional. }\end{array}$ & $\begin{array}{c}\text { Alfa } \\
\text { Cronbach }\end{array}$ \\
\hline $\begin{array}{l}\text { 1.3. Escala de Comportamentos de } \\
\text { Redesenho no Trabalho - CRT (Brasil) } \\
\text { Chinelato, Ferreira \& Valentini (2015) }\end{array}$ & $\mathrm{x}$ & $\begin{array}{l}\text { Engajamento no trabalho, desempenho } \\
\text { intrapapel, afetos positivos no trabalho, } \\
\text { capital psicológico positivo, afeto negativo e } \\
\text { neuroticismo. }\end{array}$ & $\begin{array}{c}\text { Alfa } \\
\text { Cronbach }\end{array}$ \\
\hline $\begin{array}{l}\text { 1.4. Job Crafting Scale - JCS (Itália) } \\
\text { Cenciotti, Borgogni, Callea, Colombo, } \\
\text { Cortese, Ingusci, \& Zito (2016) }\end{array}$ & $\mathrm{x}$ & $\begin{array}{l}\text { Autoeficácia no trabalho, engajamento } \\
\text { no trabalho e desempenho (avaliação de } \\
\text { efetuada pelo supervisor). }\end{array}$ & $\begin{array}{c}\text { Alfa } \\
\text { Cronbach }\end{array}$ \\
\hline $\begin{array}{l}\text { 1.5. Job Crafting Scale - JCS-J (Japão) } \\
\text { Eguchi, Shimazu, Bakker, Tims, } \\
\text { Kamiyama, Hara, \& Kawakami (2016) }\end{array}$ & $\mathrm{x}$ & $\begin{array}{l}\text { Demandas e recursos do trabalho, engajamento } \\
\text { no trabalho, satisfação no trabalho, queixas } \\
\text { físicas, sofrimento psicológico. }\end{array}$ & $\begin{array}{c}\text { Alfa } \\
\text { Cronbach }\end{array}$ \\
\hline $\begin{array}{l}\text { 2.0. Job Crafting Questionnaire - JCRQ } \\
\text { (Dinamarca) } \\
\text { Nielsen \& Abildgaard (2012) }\end{array}$ & $\mathrm{x}$ & $\begin{array}{l}\text { Engajamento no trabalho, satisfação no } \\
\text { trabalho e burnout. }\end{array}$ & $\begin{array}{l}\text { Alfa Cronbach } \\
\text { Estabilidade }\end{array}$ \\
\hline
\end{tabular}


Tabela 3 (continuação)

Qualidades Psicométricas das Medidas de Job Crafting nos Estudos Encontrados

\begin{tabular}{|c|c|c|c|}
\hline Escala - Autoria - País & $\begin{array}{l}\text { Estrutura } \\
\text { interna }\end{array}$ & $\begin{array}{l}\text { Fontes de evidências de validade } \\
\text { Relação com variáveis externas }\end{array}$ & $\begin{array}{l}\text { Fontes de } \\
\text { precisão }\end{array}$ \\
\hline $\begin{array}{l}\text { 2.1. Job Crafting Questionnaire - JCRQ } \\
\text { (Espanha, Reino Unido, Taiwan, China) } \\
\text { Nielsen, Antino, Sanz-Vergel, \& } \\
\text { Rodríguez-Muñoz (2017) }\end{array}$ & $\mathrm{x}$ & $\begin{array}{l}\text { Satisfação no trabalho e desempenho } \\
\text { (in-role, extra-role performance) }\end{array}$ & $\begin{array}{l}\text { Alfa Cronbach } \\
\text { Estabilidade }\end{array}$ \\
\hline $\begin{array}{l}\text { 3.0. Job Crafting Questionnaire (JCQ) } \\
\text { (Austrália) } \\
\text { Slemp \& Vella-Brodrick, } 2013\end{array}$ & $\mathrm{x}$ & $\begin{array}{l}\text { Comportamento pró-social no trabalho, } \\
\text { forças, satisfação no trabalho, entusiasmo } \\
\text { no trabalho, afeto positivo e afeto negativo. }\end{array}$ & $\begin{array}{c}\text { Alfa } \\
\text { Cronbach }\end{array}$ \\
\hline $\begin{array}{l}\text { 3.1. Escala de Ações de Redesenho } \\
\text { no Trabalho - EART. (Brasil) } \\
\text { Pimenta de Devotto \& Machado (2017) }\end{array}$ & $\mathrm{x}$ & Não foi realizado. & $\begin{array}{l}\text { Alfa Cronbach } \\
\text { Fidedignidade } \\
\text { composta }\end{array}$ \\
\hline $\begin{array}{l}\text { 4.0. Job Crafting Scale (Alemanha) } \\
\text { Niessen, Weseler, \& Kostova (2016) }\end{array}$ & $\mathrm{x}$ & $\begin{array}{l}\text { Iniciativa pessoal, comportamento de cidadania } \\
\text { organizacional, autoeficácia, autonomia, interde- } \\
\text { pendência de tarefas, experiência de trabalho. }\end{array}$ & $\begin{array}{c}\text { Alfa } \\
\text { Cronbach }\end{array}$ \\
\hline
\end{tabular}

A seguir serão descritas as qualidades psicométricas dos quatro instrumentos originais e das sete escalas que foram adaptadas e validadas para outros contextos.

1.0. Job Crafting Scale - JCS (Tims et al., 2012). A Job Crafting Scale - JCS (Tims, Bakker, \& Derks; 2012) operacionalizou o construto do job crafting dentro do modelo de Recursos e Demandas no Trabalho - RDT (Bakker \& Demerouti, 2007), propondo avaliar o construto por meio de três tipos de comportamentos de redesenho do trabalho: aumento dos recursos do trabalho, aumento das demandas de trabalho desafiadoras e diminuição das demandas de trabalho de obstáculo (Tims \& Bakker, 2010). A JCS foi validada em três estudos conduzidos na Holanda $(N=1,181)$. Os resultados das análises fatoriais exploratórias levaram a um instrumento final composto de 21 itens, distribuídos em quatro dimensões, ao invés dos três fatores originalmente concebidos por Tims e Bakker (2010). A dimensão "aumento dos recursos do trabalho" foi dividida em duas: "aumento dos recursos estruturais do trabalho" (e.g., autonomia, oportunidades de desenvolvimento e aprendizagem, variedade de tarefas) e "aumento dos recursos sociais do trabalho" (e.g., busca por feedback, coaching, suporte dos colegas). Os quatro fatores apresentaram índices de fidedignidade satisfatórios.

Com relação a fonte de evidências baseadas na relação com variáveis externas, o estudo evidenciou correlações positivas (baixa à moderada) com a personalidade proativa e a iniciativa pessoal. Observou-se correlações negativas com cinismo (dimensão do burnout) e as três primeiras dimensões da Job Crafting Scale, orientadas ao aumento de recursos e demandas. A validade de critério foi averiguada em relação às avaliações de colegas sobre o engajamento e desempenho no trabalho, evidenciando correlações nas direções esperadas teoricamente (Tims et al., 2012). Os resultados desta revisão indicaram que a Job Crafting Scale (Tims et al., 2012) foi adaptada e validada para cinco países: África do Sul, Alemanha, Brasil, Itália e Japão, como apresentado a seguir.

1.1 África do Sul - Job Crafting Scale (de Beer, Tims, \& Bakker, 2016). O estudo de validação da JCS (Tims et al., 2012) para o contexto sul-africano foi realizado com a participação de trabalhadores $(N=470)$ da indústria da mineração e manufatura. A estrutura de quatro fatores da escala original (JCS, Tims et al., 2012) não foi replicada para esse contexto, devido à ausência de discriminação satisfatória entre dois fatores (aumento dos recursos estruturais e aumento das demandas desafiadoras), o que levou os autores à combinarem ambos fatores em um novo fator denominado "aumento dos recursos estruturais e das demandas desafiadoras". A escala final composta de três dimensões apresentou índices de consistência interna satisfatórios em ambos grupos de trabalhadores. O aumento dos recursos estruturais e das demandas desafiadoras e o aumento dos recursos sociais foram preditores do engajamento do trabalho de trabalhadores na mineração e na manufatura. O aumento dos recursos sociais foi um preditor significativo da satisfação no trabalho em ambas indústrias. A diminuição das demandas de obstáculo foi preditor negativo da satisfação no trabalho de empregados da mineração (de Beer et al., 2016).

1.2. Alemanha - Job Crafting Scale (Lichtenthaler \& Fischbach, 2016). A JCS (Tims et al., 2012) foi validada para a Alemanha em dois estudos independentes $(N=512)$ com policiais. O Estudo $1(n=243)$ confirmou a estrutura de quatro fatores da escala original com índices de confiabilidade satisfatórios. A iniciativa pessoal foi preditora positiva das três dimensões do job crafting consideradas promotoras de desenvolvimento e bem-estar (promotion-focused job crafting): aumentar recursos estruturais, recursos sociais, demandas desafiadoras. A 
inciativa pessoal foi preditora negativa da diminuição das demandas de obstáculo, considerada pelos autores como uma dimensão preventiva (prevention-focused job crafting), cujo foco de ação de redesenho é a segurança e proteção no trabalho. O Estudo $2(n=269)$ encontrou evidências que as três dimensões com foco na promoção (promotion-focused job crafting) foram preditoras positivas significativas do engajamento no trabalho e negativas da exaustão emocional. A diminuição das demandas de obstáculo não foi preditora do engajamento no trabalho, porém foi positiva da exaustão emocional (Lichtenthaler \& Fischbach, 2016).

1.3. Brasil - Escala de Comportamentos de Redesenho no Trabalho - CRT (Chinelato, Ferreira, \& Valentini, 2015). A versão brasileira da Job Crafting Scale, nomeada Escala de Comportamentos de Redesenho no Trabalho - CRT (Chinelato, Ferreira, \& Valentini, 2015), reuniu evidências de validade $(N=491)$ por meio de análises fatoriais confirmatórias que revelaram o melhor ajuste de um modelo alternativo de três fatores de primeira ordem (aumento dos recursos estruturais, dos recursos sociais, das demandas desafiadoras) com um fator geral de segunda ordem. A quarta dimensão "diminuição das demandas de obstáculo" da escala original foi excluída por não apresentar carga fatorial satisfatória. Portanto, as evidências de validade baseadas na estrutura interna obtidas não corroboraram o modelo de quatro fatores da escala original (Chinelato et al., 2015).

As fontes de evidências baseadas nas relações da CTR com variáveis externas, revelaram correlações positivas com o engajamento no trabalho, com o desempenho intrapapéis de trabalho, com os afetos positivos no trabalho e com o capital psicológico positivo. Obteve-se também correlações negativas baixas entre aumento dos recursos estruturais com os afetos negativos no trabalho e o neuroticismo. Correlações negativas baixas também foram observadas entre o aumento das demandas desafiadoras e o neuroticismo (Chinelato et al., 2015).

\subsection{Itália - Job Crafting Scale - Italian Version} (Cenciotti et al., 2016). O estudo italiano $(N=721)$ com duas amostras independentes, reuniu evidências de validade baseadas na estrutura interna e na relação com variáveis externas. Testou-se apenas as três dimensões da JCS (Tims et al., 2012), orientadas na direção positiva de "aumentar": os recursos estruturais do trabalho, os recursos sociais do trabalho e as demandas de trabalho desafiadoras. A dimensão "diminuição das demandas de obstáculo" foi deliberadamente excluída do estudo. Conduziu-se uma análise fatorial exploratória $(n=311)$ e uma análise fatorial confirmatória $(n=410)$. Confirmou-se a estrutura de três fatores (aumento dos recursos estruturais; aumento dos recursos sociais; aumento das demandas desafiadoras) com a exclusão de dois itens, resultando em uma escala de 13 itens com índices de confiabilidade satisfatórios. O aumento dos recursos estruturais do trabalho e das demandas desafiadoras apresentaram correlações positivas moderadas com a autoeficácia no trabalho e o engajamento no trabalho, ao passo que o aumento de recursos sociais no trabalho apresentou correlações positivas fracas com ambas variáveis. Correlações positivas fracas foram encontradas com relação ao desempenho no trabalho, avaliado pelos supervisores dos participantes (Cenciotti et al., 2016).

1.5. Japão - Job Crafting Scale (JCS-J) (Eguchi et al., 2016). A versão japonesa (JCS-J) foi validada utilizando uma amostra $(N=972)$ on-line com funcionários de uma empresa manufatureira. Contrariamente ao modelo original, a estrutura de cinco fatores foi a que melhor explicou a covariância entre os itens e os dados da amostra. A dimensão diminuição das demandas de obstáculo foi dividida em duas, sugerindo uma dimensão relacionada à diminuição das demandas emocionais e outra às demandas cognitivas. Porém os autores decidiram adotar o modelo original de quatro fatores, dado que seus índices de ajuste foram aceitáveis e para poder facilitar as comparações dos achados com outras pesquisas (Eguchi et al., 2016).

As fontes de evidências baseadas nas relações com variáveis externas foram investigadas em relação as demandas e recursos do trabalho. O aumento das demandas de trabalho desafiadoras correlacionou-se positivamente com as demandas de trabalho desafiadoras, indicando que este tipo de comportamento de redesenho do trabalho elevaria os níveis de carga de trabalho e pressão de tempo. Observou-se correlações positivas significativas entre o aumento dos recursos estruturais e sociais e a disponibilidade de ambos no trabalho. Esse achado sugere que os indivíduos que efetivamente empregaram ações de redesenho para aumentar os recursos estruturais e sociais do trabalho estariam mais propensos a relatar níveis mais elevados de ambos recursos (Eguchi et al., 2016).

Observou-se correlações significativas entre as três dimensões (aumento dos recursos estruturais, aumento dos recursos sociais, aumento das demandas desafiadoras) com o engajamento no trabalho e a satisfação no trabalho. A diminuição das demandas de trabalho não apresentou correlações com o engajamento no trabalho e esteve negativamente associada com a satisfação no trabalho. O aumento dos recursos estruturais do trabalho obteve correlações negativas com outros desfechos negativos do bem-estar (e.g., queixas físicas e sofrimento psicológico), ao passo que a diminuição das demandas de trabalho correlacionou-se de forma positiva com esses índices. O estudo confirmou que JCS-J é uma medida adequada para avaliar o job crafting no contexto japonês (Eguchi et al., 2016). 
2.0. Job Crafting Questionnaire for Blue-Collar Workers - JCRQ (Nielsen \& Abildgaard, 2012). Com base na JCS (Tims et al., 2012), Nielsen e Abildgaard (2012) adaptaram uma medida de job crafting para ser utilizada com trabalhadores operacionais em contextos de baixa autonomia e controle no trabalho. Argumentou-se que comportamentos de redesenho associados à dimensão de "aumento dos recursos estruturais" (e.g., iniciar novos projetos) envolveriam altos níveis de tomada de decisão e poderiam não ser relevantes em contextos operacionais. Criaram-se novos itens para capturar comportamentos de job crafting de trabalhadores operacionais. A validade e precisão da escala foram testadas em um estudo longitudinal com trabalhadores do serviço de entrega do correio na Dinamarca ( $n=362$ no T1, $n=408$ no T2). Resultados confirmaram a existência de cinco dimensões (15 itens) com índices de confiabilidade satisfatórios: aumento das demandas desafiadoras, diminuição das demandas sociais, aumento dos recursos sociais no trabalho, aumento das demandas quantitativas e diminuição das demandas de obstáculo. A JCRQ apresentou estabilidade e coeficientes de correlação entre as dimensões no T1 e T2 satisfatórios. Esse estudo ampliou a utilização de medidas de job crafting para demonstrar que trabalhadores operacionais se engajam em comportamentos de redesenho do trabalho. Entretanto, o job crafting não foi um forte preditor de desfechos do bem-estar ocupacional, tanto positivos (e.g., engajamento do trabalho e satisfação no trabalho), como negativos (e.g., burnout).

2.1. China, Taiwan, Espanha e Reino Unido-Job Crafting Questionnaire for Blue-Collar Workers - JCRQ (Nielsen \& Abildgaard, 2012). Posteriormente a validade e precisão da JCRQ (Nielsen \& Abildgaard, 2012) foram testadas em quatro estudos com amostras múltiplas e utilizando diferentes métodos (Nielsen, Antino, Sanz-Vergel, \& Rodríguez-Muñoz, 2017). A estrutura de cinco fatores foi confirmada na China $(n=170)$, Taiwan $(\mathrm{n}=165)$, Espanha $(n=164)$ e Reino Unido $(n=109)$. $\mathrm{O}$ instrumento demonstrou estabilidade em um estudo de longitudinal realizado com trabalhadores espanhóis de uma empresa de serviço de limpeza $(n=191)$. Confirmou-se também a validade do instrumento com itens reformulados para capturar as experiências diárias de job crafting e sua aplicabilidade em um estudo com medidas diárias (diary studies) (Espanha, $n=164$ ), que permitiram avaliar as oscilações diárias de comportamentos de job crafting. Evidências de validade baseadas na relação com variáveis externas foram encontradas com medidas de bem-estar (e.g., satisfação no trabalho) e desempenho (in-role lextra-role performance) (Nielsen, Antino, SanzVergel, \& Rodríguez-Muñoz, 2017).

3.0. Job Crafting Questionnaire - JCQ (Slemp \& Vella-Brodrick, 2013). Diferentemente das escalas descritas anteriormente, o Job Crafting Questionnaire - JCQ
(Slemp \& Vella-Brodrick, 2013) está baseado na formulação teórica seminal do construto e avalia simultaneamente três dimensões: as ações de redesenho da tarefa (task crafting), as reformulações cognitivas (cognitive crafting) e as ações de redesenho das relações (relational crafting) (Wrzesniewski \& Dutton, 2001). A análise fatorial exploratória e confirmatória do instrumento JCQ demonstrou que a estrutura de três fatores foi a mais parcimoniosa para explicar o conjunto dos 15 itens do instrumento. Os índices de consistência interna foram satisfatórios.

Com relação as fontes de evidências baseadas na relação com variáveis externas, corroborou-se a correlação positiva do JCQ com índices de comportamento proativo (e.g., comportamento pró-social no trabalho e uso de forças) e com o funcionamento positivo no trabalho (e.g., satisfação no trabalho, entusiasmo no trabalho e afeto positivo). O JCQ esteve negativamente associado ao afeto negativo (Slemp \& Vella-Brodrick, 2013). De acordo com esta revisão, o JCQ (Slemp \& Vella-Brodrick, 2013) foi adaptado apenas para o contexto brasileiro.

\subsection{Brasil - Escala de Ações de Redesenho do} Trabalho - EART (Pimenta de Devotto, 2016). A versão brasileira da JCQ (Slemp \& Vella-Brodrick, 2013) foi denominada de Escala de Ações de Redesenho do Trabalho - EART. Suas propriedades psicométricas foram investigadas em dois estudos, sendo o primeiro com uma amostra on-line $(n=261)$ e o segundo $(n=152)$ com funcionários de uma organização privada do interior paulista. Os resultados de ambos os estudos corroboram a estrutura interna do instrumento por meio de técnicas exploratórias (análise fatorial exploratória e análise de rede) e análise fatorial confirmatória. $\mathrm{Na}$ análise fatorial exploratória do Estudo 1, os três fatores (redesenho da tarefa, reformulação cognitiva e redesenho das relações) estiveram significativamente associados e seus índices de consistência interna foram satisfatórios. A dimensão cognitiva apresentou também maior variância explicada $(16,81 \%)$ em relação às respostas da amostra do Estudo 1 , quando comprada às dimensões da tarefa $(10 \%)$ e das relações $(7,84 \%)$.

As evidências de validade da estrutura interna foram obtidas por meio da análise fatorial confirmatória (AFC) no Estudo 2, que confirmou a estrutura de três fatores encontrada no Estudo $1 \mathrm{em}$ uma amostra independente. Os índices de fidedignidade composta das três subescalas foram satisfatórias. Da mesma forma, a variância média extraída dos itens de cada um dos fatores foram respectivamente, $\operatorname{tarefa}=45 \%$, cognitiva $=73 \%$ e relações $=39 \%$. Em ambos estudos, destacou-se a saliência da dimensão cognitiva em termos da sua primazia na extração de fatores, da sua variância explicada e da sua confiabilidade.

4.0. Job Crafting Scale (Niessen, Weseler, \& Kostova, 2016). A Job Crafting Scale (Niessen, Weseler \& Kostova, 2016) propôs uma forma de avaliação 
alinhada com a formulação teórica seminal do construto (Wrzesniewski \& Dutton, 2001). Assim como Slemp e Vella Brodrick (2013), as autoras enfatizaram a importância de aferir a dimensão cognitiva do construto, porque a reformulação cognitiva é uma estratégia proativa importante na transação indivíduo-ambiente. O indivíduo ressignifica o próprio trabalho e pode construir uma identidade mais positiva nele (Niessen et al., 2016).

As evidências de validade e precisão do instrumento foram avaliadas em dois estudos. No Estudo $1(n=466)$, avaliou-se com amostras independentes, a estrutura interna do instrumento por meio de análises fatoriais exploratória $(n=233)$ e confirmatória $(n=233)$. Os resultados confirmaram a estrutura de três fatores (task crafting, cognitive crafting, relational crafting) que explicaram $65,54 \%$ da variância dos itens. A consistência interna e a fidedignidade das subescalas foram satisfatórias. Agregou-se evidências de validade convergente e discriminante em relação à variáveis externas do comportamento proativo (e.g., iniciativa pessoal e comportamento organizacional de cidadania).

O Estudo 2 ( $n=118$, com dois tempos de medida) avaliou a relação do job crafting com os antecedentes motivacionais (e.g., autoeficácia, necessidade de conexão social e necessidade de uma autoimagem positiva) e com dois tipos de antecedentes distais: as características do trabalho (e.g., autonomia, interdependência de tarefas) e as diferenças individuais (experiência de trabalho). A necessidade de autoimagem (Tempo 1) foi a principal preditora das ações de redesenho no trabalho (Tempo 2), seguida da experiência de trabalho. A necessidade de conexão humana (Tempo 1) relacionou-se com o job crafting (Tempo 2) quando os índices de autoeficácia foram elevados. A necessidade de conexão humana e autoeficácia não foram preditores independentes do job crafting, mas sua ação conjunta demonstrou que necessidade de conexão humana influenciou o redesenho das relações (relational crafting) quando os indivíduos eram autoeficazes. Obteve-se evidências de que o job crafting correlacionou-se positivamente com o índice de ajuste indivíduo-trabalho (person-job fit) (Niessen et al., 2016).

\section{Discussão}

Desde o surgimento do job crafting, em 2001, na pesquisa qualitativa, o número de pesquisas sobre o construto vem aumentando consideravelmente e esse ritmo acelera-se a com o surgimento de instrumentos com qualidade científica para a operacionalização do construto (Pimenta de Devotto \& Machado, 2017). Essa revisão sobre as medidas de job crafting demonstrou que os pesquisadores contam até a presente data, com ao menos quatro opções para avaliar o construto. Dois tipos de medidas foram operacionalizados de acordo com o modelo RDT (Bakker \& Demerouti, 2007; Tims \& Bakker, 2010): a JCS (Tims et al., 2012) e o JCRQ (Nielssen et al., 2012), sendo esta última adequada para contextos de trabalho operacional. Outras duas medidas, a JCQ (Slemp \& Vella-Brodrick, 2013) e a JCS (Niessen et al., 2016), utilizaram a formulação teórica de Wrzesniewski e Dutton (2001) (Tabela 1).

A Job Crafting Scale (Tims et al., 2012) tem sido o instrumento mais utilizado em estudos empíricos quantitativos (Pimenta de Devotto \& Machado, 2017) e, de acordo com os resultados desta revisão, está disponível em vários idiomas: inglês (Tims et al., 2012; de Beer et al., 2016), português brasileiro (Chinelato et al., 2015), italiano (Cenciotti et al., 2016), japonês (Eguchi et al., 2016) e alemão (Lichtenthaler \& Fischbach, 2016). Sabe-se que a JCS (Tims et al., 2012) foi adaptada também para a Turquia e a Espanha (Nielsen et al., 2016) e para o Irã e os Estados Unidos da América (Lichtenthaler \& Fischbach, 2016), porém esses estudos não foram recuperados pelas bases de dados utilizadas nesta revisão. A JCS (Tims et al., 2012) enfatizou os comportamentos que geram mudanças tangíveis nas tarefas e nas relações e desconsiderou as mudanças cognitivas intangíveis que influenciam essas ações (Slemp \& Vella-Brodrick, 2013; Niessen et al., 2016).

Do ponto de vista da qualidade psicométrica das 11 medidas avaliadas nesta revisão, observa-se que as estratégias preponderantes nos estudos de construção das escalas e nos estudos de adaptação e validação destas para outros contextos, foram a busca por fontes de evidências de validade baseadas na estrutura interna e na relação do job crafting com seus antecedentes individuais (e.g., variáveis de recursos pessoais e personalidade proativa) e contextuais (e.g., recursos e demandas no trabalho), bem como com seus desfechos (e.g., engajamento no trabalho, afetos, burnout). Em relação à precisão, os estudos reportam evidências principalmente por meio do alfa de Cronbach. Somente dois estudos apresentaram evidências de precisão por estabilidade. Não foram encontradas informações relacionadas às normas das escalas em nenhum estudo. Portanto, sugere-se ampliar a busca por evidências de precisão em termos da estabilidade dessas medidas, bem como oferecer normas de comparação para avaliação do job crafting, considerando a importância da amostra. Observa-se também que todos os 11 estudos selecionados utilizaram a coleta de dados com escalas disponibilizadas on-line, o que reflete a tendência da administração on-line como procedimento escolhido principalmente na área organizacional (Elosua, 2017).

Com relação a replicabilidade da estrutura fatorial dos instrumentos adaptados, observa-se que, dos sete estudos de adaptação, apenas dois estudos (Chinelato et al., 2015; De Beer et al., 2016) não replicaram a estrutura fatorial do instrumento avaliado, o Job Crafting Scale - JCS (Tims et al., 2012). A falta de replicabilidade da estrutura fatorial não se deve ao uso de métodos de estimação de parâmetro inadequados, dado que o Weighted Least Squares Mean and Variance Adjusted (WLSMV) foi empregado em ambos estudos. No estudo de adaptação da JCS (Tims et al., 2012) para o contexto brasileiro, a 
subescala "diminuição das demandas de obstáculo" não foi replicada para a amostra brasileira. Tal fato, segundo Chinelato et al. (2015), pode ser explicado por dois fatores: 1. a baixa escolaridade da amostra brasileira, que teria interferido nas percepções dos trabalhadores menos qualificados acerca das demandas de trabalho que deveriam ser redesenhadas; e 2. as relações de trabalho marcadas por forte centralização de poder na liderança, o que seria um obstáculo à autonomia dos trabalhadores. No caso da validação transcultural para a África do Sul, a ausência de validade discriminante entre "aumentando os recursos estruturais do trabalho" e "aumentando as demandas desafiadoras", levou a solução de combinar ambos fatores em único fator. De acordo com De Beer e colegas (2016), tal ausência de validade discriminante pode ter sido decorrente da interpretação dos itens em relação à linguagem utilizada em inglês. Essas limitações metodológicas indicam que os pesquisadores devem estar atentos à seleção de suas amostras, às possíveis diferenças culturais, bem como à adequação da linguagem utilizada na construção dos itens à cultura local.

Observa-se ainda que, dos sete estudos de adaptação transcultural das medidas de job crafting, apenas um estudo (Nielsen et al., 2017) investigou a invariância de medida do instrumento JCRQ (Nielsen \& Abildgaard, 2012), em quatro países (i.e., China, Espanha, Taiwan e Reino Unido), por meio da Análise Fatorial Confirmatória Multigrupo (AFCMG). Tal técnica permite avaliar em que medida a configuração e os parâmetros do instrumento são invariantes para diferentes populações simultaneamente (Damásio, 2013), ou seja, até que ponto os respondentes de diferentes culturas interpretam a medida de maneira conceitualmente similar. Nielsen et al. (2017) testaram três modelos de invariância (i.e., invariância configural, invariância métrica e invariância escalar) do JCRQ por meio comparações de amostras cruzadas. Constatou-se invariância configural na comparação das amostras dos quatro países e invariância métrica entre respondentes do Reino Unido, Espanha e Twaian (Nielsen et al., 2017). Apesar da avaliação da invariância de medida ser considerada um aspecto crucial para a validação de instrumentos em estudos transculturais e a AFCMG um importante recurso no refinamento de medidas (Damásio, 2013), constatou-se uma baixa produção de estudos de validação transcultural de medidas de job crafting com condução da AFCMG. Tal ocorrência pode ser devido à dificuldade de acesso aos dados para a comparação de amostras de diferentes países. Portanto, existem oportunidades de pesquisas futuras para avaliação da invariância de medida das medidas de job crafting a partir da colaboração entre pesquisadores no compartilhamento de suas amostras.

Concluí-se que o construto do job crafting é mensurável de forma válida e precisa. As diferentes medidas de job crafting seguem os padrões internacionais de validade e precisão e representam um importante avanço nos recursos metodológicos para poder alcançar objetivos de pesquisa mais amplos. A escolha da medida de job crafting dependerá dos objetivos do pesquisador e da conceitualização do construto adotada. Provavelmente, trabalhos empíricos com enquadramento teórico no modelo RDT (Bakker \& Demerouti, 2007) operacionalizem o job crafting em termos de comportamentos de redesenho orientados à otimização de recursos e demandas no trabalho e, por essa razão, optem por avaliá-lo com a JCS (Tims et al., 2012) ou com a JCRQ (Nielsen et al., 2016). Estudos que busquem investigar mudanças no significado do trabalho e na construção de identidades mais positivas nele poderão formular o job crafting como mudanças tangíveis nas características do trabalho e mudanças intangíveis nas percepções sobre o trabalho (Lichtenthaler \& Fischbach, 2016). Estudos nessa perspectiva provavelmente optem pelo JCQ (Slemp \& Vella-Brodrick, 2013) ou pela JCS (Niessen et al., 2016).

Pesquisadores brasileiros podem contar com duas medidas adaptadas e validadas para esse contexto, sendo que ambas medem o job crafting de maneira qualitativamente diferente. A Escala de Comportamentos de Redesenho no Trabalho (Chinelato et al., 2015) operacionalizou o construto de acordo com a formulação teórica de Tims e Bakker (2010). A Escala de Ações de Redesenho no Trabalho (Pimenta de Devotto, 2016) operacionalizou o job crafting de acordo com o modelo teórico Wrzesniewski e Dutton (2001). Conclui-se que existem bases psicométricas para verificar o desenvolvimento de ações de redesenho no trabalho no contexto brasileiro por meio de pesquisas de intervenção que avaliem o efeito dessas ações para indivíduos e organizações.

Dado a complexidade do contexto de trabalho no mundo, marcado pelo aumento da incerteza e da interdependência entre as funções (Grant \& Parker, 2009) e pela necessidade de adaptar e inovar continuamente (Janssen, Van de Vliert, \& West, 2004), a perspectiva do job crafting ganha proeminência ao enfatizar o papel ativo dos indivíduos na construção do seu próprio trabalho. A pesquisa empírica em job crafting poderá fazer promissoras contribuições para ajudar a construir um mundo com trabalhadores mais autônomos, engajados, produtivos, inovadores e saudáveis.

\section{Referências}

American Educational Research Association, American Psychological Association \& National Council on Measurement in Education (2014). Standards for educational and psychological testing. Washington, DC: American Educational Research Association. 
Bakker, A. B. (2015). Top-down and bottom-up interventions to increase work engagement. Em Hartung, P. J., Savickas, M. L., \& Walsh, B. W (Eds.), APA Handbook of Career Intervention, vol. 2. Applications (pp. 427-38). Washington, DC: American Psychological Association.

Bakker, A. B., \& Demerouti, E. (2007). The job demands-resources model: State of the art. Journal of Managerial Psychology, 22(3), 309-328. doi: 10.1108/02683940710733115

Berg, J. M., Dutton, J. E., \& Wrzesniewski, A. (2008). What is job crafting and why does it matter. From the Center for Positive Organizational Scholarship, 15, 1-8. Recuperado de http://positiveorgs.bus.umich.edu/wp-content/uploads/What-is-Job-Crafting-and-Why-Does-itMatter1.pdf

Berg, J. M., Grant, A. M., \& Johnson, V. (2010). When callings are calling: Crafting work and leisure in pursuit of unanswered occupational callings. Organization Science, 21(5), 973-994. doi: 10.1287/orsc.1090.0497

Berg, J. M., Wrzesniewski, A., \& Dutton, J. E. (2010). Perceiving and responding to challenges in job crafting at different ranks: When proactivity requires adaptively. Journal of Organizational Behavior, 31(2-3), 158-186. doi: 10.1002/job.645

Chinelato, R. S. D. C., Ferreira, M. C., \& Valentini, F. (2015). Evidence of validity of the job crafting behaviors scale. Paidéia, 25(62), 325332. doi: 10.1590/1982-43272562201506

Cenciotti, R., Borgogni, L., Callea, A., Colombo, L., Cortese, C. G., Ingusci, E., ... \& Zito, M. (2016). The Italian version of the Job Crafting Scale (JCS). Applied Psychology Bulletin, 64(277), 28-36. Recuperado de https://ueaeprints.uea.ac.uk/62994/

Damásio, B. F. (2013). Contribuições da Análise Fatorial Confirmatória Multigrupo (AFCMG) na avaliação de invariância de instrumentos psicométricos. Psico-USF, 18(2), 211-220. doi: 10.1590/S1413-82712013000200005

De Beer, L. T., Tims, M., \& Bakker, A. B. (2016). Job crafting and its impact on work engagement and job satisfaction in mining and manufacturing. South African Journal of Economic and Management Sciences, 19(3), 400-412. doi: 10.17159/2222-3436/2016/v19n3a7

Demerouti, E. (2014). Design your own job through job crafting. European Psychologist, 19(4), 237-247. doi: 10.1027/1016-9040/a00018888

Eguchi, H., Shimazu, A., Bakker, A. B., Tims, M., Kamiyama, K., Hara, Y., \& Kawakami, N. (2016). Validation of the Japanese version of the job crafting scale. Journal of occupational health, 58(3), 231-240. doi: 10.1539/joh.15-0173-OA

Elosua, P. (2017). Avances, proyectos y retos internacionales ligados al uso de tests en Psicología. Estudos de Psicologia. (Campinas), 34(2), 201210. doi: 10.1590/1982-02752017000200002

Geisinger, K. (2013). APA Handbook on Psychological Assessment. vol. I, pp.21-42. Washington, DC: American Psychological Association.

Grant, A. M., \& Parker, S. K. (2009). 7 redesigning work design theories: The rise of relational and proactive perspectives. The Academy of Management Annals, 3(1), 317-375. doi: 10.1080/19416520903047327

Janssen, O., Van de Vliert, E., \& West, M. (2004). The bright and dark sides of individual and group innovation: A special issue introduction. Journal of Organizational Behavior, 25(2), 129-145. doi: 10.1002/job.242

Lichtenthaler, P. W., \& Fischbach, A. (2016). The conceptualization and measurement of job crafting. Zeitschrift für Arbeits-und Organisationspsychologie A\&O, 60, 173-186. doi: 10.1026/0932-4089/a000219

Nielsen, K.; Abildgaard, J. S. (2012). The development and validation of a job crafting measure for use with blue-collar workers. Work \& Stress: An International Journal of Work, Health and Organizations, 26(4), 365-384. doi:10.1080/02678373.2012.733543

Nielsen, K., Antino, M., Sanz-Vergel, A., \& Rodríguez-Muñoz, A. (2017). Validating the Job Crafting Questionnaire (JCRQ): A multimethod and multi-sample study. Work \& Stress, 31(1), 82-99. doi: 10.1080/02678373.2012.733543

Niessen, C., Weseler, D., \& Kostova, P. (2016). When and why do individuals craft their jobs? The role of individual motivation and work characteristics for job crafting. Human Relations, 69(6), 1287-1313. doi: 10.1177/0018726715610642

Petrou, P., Demerouti, E., Peeters, M. C. W., Schaufeli, W. B., \& Hetland, J. (2012). Crafting a job on a daily basis: Contextual correlates and the link to work engagement. Journal of Organizational Behavior, 33(8), 1120-1141. doi: 10.1002/job.1783

Pimenta de Devotto, R. \& Machado. (2017). Job crafting: Uma revisão da produção científica internacional. Psico-USF, 22(3), 413-423. doi: $10.1590 / 1413-82712017220303$

Pimenta de Devotto, R. (2016). Adaptação e validação do Questionário de Job Crafting e sua relação com estados positivos no trabalho (Dissertação de mestrado). Recuperado de http://bancodeteses.capes.gov.br

Rudolph, C. W., Katz, I. M., Lavigne, K. N., \& Zacher, H. (2017). Job crafting: A meta-analysis of relationships with individual differences, job characteristics, and work outcomes. Journal of Vocational Behavior, 102(2017), 112-138. doi: 10.1016/j.jvb.2017.05.008

Slemp, G. R., \& Vella-Brodrick, D. A. (2013). The Job Crafting Questionnaire: A new scale to measure the extent to which employees engage in job crafting. International Journal of Wellbeing, 3(2), 126-146. doi: 10.5502/ijw.v3i2.1

Tims, M., \& Bakker, A. B. (2010). Job crafting: Towards a new model of individual job redesign. SA Journal of Industrial Psychology, 36(2), 1-9. doi: 10.4102/ sajip.v36i2.841

Tims, M., Bakker, A. B., \& Derks, D. (2012). Development and validation of the job crafting scale. Journal of Vocational Behavior, 80(1), 173186. doi:10.1016/j.jvb.2011.05.009

Tims, M., Bakker, A. B., \& Derks, D. (2014). Daily job crafting and the self-efficacy -Performance relationship. Journal of Managerial Psychology, 29(5), 490-507. doi: 10.1108/JMP-05-2012-0148

Wrzesniewski, A., \& Dutton, J. E. (2001). Crafting a job: Revisioning employees as active crafters of their work. Academy of Management Review, 26(2), 179-201. doi:10.5465/AMR.2001.4378011

Wrzesniewski, A., LoBuglio, N., Dutton, J. E., \& Berg, J. M. (2013). Job crafting and cultivating positive meaning and identity in work. Advances in Positive Organizational Psychology, 1(1), 281-302. doi: 10.1108/S2046-410X(2013)0000001015

\section{Sobre as autoras}

Rita Pimenta de Devotto é pedagoga (Unicamp), com MBA Executivo pelo IAE Business School (Argentina), Master of Science in Creativity and Problem Solving, State University of New York (EUA), mestre em Psicologia (PUCAMP), doutoranda do Programa de Pós-Graduação em Psicologia da PUC - Campinas e professora convidada do IAE Business School (Argentina).

Solange Muglia Wechsler é psicóloga (PUC-RJ), mestre e doutora em Psicologia pela University of Georgia (EUA), pós-doutora pelo Torrance Center of Creative Studies, professora do Programa de Pós-Graduação em Psicologia da PUC - Campinas, editora chefe da revista Estudos de Psicologia (Campinas) e dirige o Laboratório de Avaliação e Medidas Psicológicas na Pontifícia Universidade Católica de Campinas. 